УДК $517.95+532$

\title{
A Hydrostatic Model for an Ideal Fluid: Group Properties of Equations and their Solutions
}

\author{
Alexander A. Rodionov* \\ Institute of Computational Modelling SB RAS \\ Akademgorodok, 50/44, Krasnoyarsk, 660036 \\ Russia \\ Institute of Mathematics and Computer Science \\ Siberian Federal University \\ Svobodny, 79, Krasnoyarsk, 660041
}

Russia

Received 07.01.2015, received in revised form 24.02.2015, accepted 27.05.2015

Group properties of hydrostatic model equations of a layer motion in an ideal fluid on a function defining the free surface and the thickness of the fluid layer under the free boundary are studied. Examples of several exact solutions in Cartesian and cylindrical coordinates are given, they determine the free surface and the pressure on it.

Keywords: ideal fluid, hydrostatic model, group analysis, exact solutions.

DOI: $10.17516 / 1997-1397-2015-8-3-320-326$

\section{Problem statement. Basic equations}

Consider equations of motion for an ideal incompressible fluid in a gravitational field

$$
\begin{gathered}
u_{t}+u u_{x}+v u_{y}+w u_{z}+\frac{1}{\rho} p_{x}=0, \quad v_{t}+u v_{x}+v v_{y}+w v_{z}+\frac{1}{\rho} p_{y}=0, \\
w_{t}+u w_{x}+v w_{y}+w w_{z}+\frac{1}{\rho} p_{z}=-g, \quad u_{x}+v_{y}+w_{z}=0 .
\end{gathered}
$$

Here $u, v, w$ are components of the velocity vector; the pressure $p$ is the function of the variables $x, y, z$ and of time $t$; the fluid density $\rho$ is constant (we can take $\rho=1$ );g= const $>0$ is the acceleration of the force of gravity which acts in the negative direction of the $z$ axis.

Let us assume that pressure in the fluid depends linearly on the depth

$$
p_{z}=-g
$$

This assumption is often used to describe processes in oceanography [1]. Then

$$
p(x, y, z, t)=-g z+q(x, y, t),
$$

where $q(x, y, z)$ is a new function. In this situation the system (1) is rewritten in the following form

$$
\begin{gathered}
u_{t}+u u_{x}+v u_{y}+w u_{z}+q_{x}=0, \quad v_{t}+u v_{x}+v v_{y}+w v_{z}+q_{y}=0, \\
w_{t}+u w_{x}+v w_{y}+w w_{z}=0, \quad u_{x}+v_{y}+w_{z}=0 .
\end{gathered}
$$

Let $z=\eta(x, y, t)$ be the equation of the free boundary on which the dynamic and kinematic conditions are fulfilled

$$
p(x, y, \eta(x, y, t), t)=p_{a}(x, y, t)
$$

aarod@icm.krasn.ru

(c) Siberian Federal University. All rights reserved 


$$
\eta_{t}+u(x, y, \eta(x, y, t), t) \eta_{x}+v(x, y, \eta(x, y, t), t) \eta_{y}=w(x, y, \eta(x, y, t), t),
$$

where $p_{a}(x, y, t)$ is the atmospheric pressure.

From formula (3) taking into consideration the condition (5) on the free surface we find that

$$
p_{a}(x, y, t)=-g \eta(x, y, t)+q(x, y, t) .
$$

Equation (7) determines uniquely the free surface via the function $q(x, y, t)$ that can be derived from the system (4).

Sometimes it is convenient to solve the equations in cylindrical coordinates. Let us rewrite the system (4) using the variables $(r, \theta, z)$

$$
\begin{gathered}
\bar{u}_{t}+\bar{u} \bar{u}_{r}+\frac{\bar{v}}{r} \bar{u}_{\theta}+\bar{w} \bar{u}_{z}-\frac{\bar{v}^{2}}{r}+q_{r}=0, \quad \bar{v}_{t}+\bar{u} \bar{v}_{r}+\frac{\bar{v}}{r} \bar{v}_{\theta}+\bar{w} \bar{v}_{z}+\frac{\bar{u} \bar{v}}{r}+\frac{1}{r} q_{\theta}=0 \\
\bar{w}_{t}+\bar{u} \bar{w}_{r}+\frac{\bar{v}}{r} \bar{w}_{\theta}+\bar{w} \bar{w}_{z}=0, \quad \bar{u}_{r}+\frac{1}{r} \bar{v}_{\theta}+\bar{w}_{z}+\frac{1}{r} \bar{u}=0 .
\end{gathered}
$$

Here $(\bar{u}, \bar{v}, \bar{w})$ are the components of the radial, azimuthal, and axial velocity dependent on $(r, \theta, z, t)$; the function $q$ from formula (3) depends on $(r, \theta, t)$.

Along with equations (4) an approximate model is also considered, which describes the motion of the fluid as $z \rightarrow \varepsilon z, w \rightarrow \varepsilon w$. In the limit, as $\varepsilon \rightarrow 0$, the system (4) assumes the following form

$$
\begin{gathered}
u_{t}+u u_{x}+v u_{y}+w u_{z}+q_{x}=0, \quad v_{t}+u v_{x}+v v_{y}+w v_{z}+q_{y}=0, \\
u_{x}+v_{y}+w_{z}=0, \quad q_{z}=0 .
\end{gathered}
$$

We perform the group analysis for systems of equations (4) and (9), find the Lie algebra of admissible operators of these systems and construct exact solutions.

\section{Group properties of the equations}

Study the group properties of equations (4). We introduce the following index notation $u^{1}=u, u^{2}=v, u^{3}=w, u^{4}=q, x^{1}=x, x^{2}=y, x^{3}=z, x^{4}=t$. In this notation equations (4), being supplemented by the requirement $q_{z}=0$, assume the following form

$$
\begin{gathered}
u_{4}^{1}+u^{1} u_{1}^{1}+u^{2} u_{2}^{1}+u^{3} u_{3}^{1}+u_{1}^{4}=0, \quad u_{4}^{2}+u^{1} u_{1}^{2}+u^{2} u_{2}^{2}+u^{3} u_{3}^{2}+u_{2}^{4}=0 \\
u_{4}^{3}+u^{1} u_{1}^{3}+u^{2} u_{2}^{3}+u^{3} u_{3}^{3}=0, \quad u_{1}^{1}+u_{2}^{2}+u_{3}^{3}=0, \quad u_{3}^{4}=0 .
\end{gathered}
$$

The lower index is the differentiation.

We find an admissible operator for the system (9) in the form

$$
X=\xi^{i}(\mathbf{x}, \mathbf{u}) \frac{\partial}{\partial x^{i}}+\eta^{k}(\mathbf{x}, \mathbf{u}) \frac{\partial}{\partial u^{k}} .
$$

Here the summation is over $i, k=1,2,3,4$. The operator prolongs to the first derivatives

$$
\underset{1}{X}=X+\varsigma_{i}^{k} \frac{\partial}{\partial u_{i}^{k}}, \quad \varsigma_{i}^{k}=\frac{\partial \eta^{k}}{\partial x^{i}}+u_{i}^{l} \frac{\partial \eta^{k}}{\partial u^{l}}-u_{j}^{k}\left(\frac{\partial \xi^{j}}{\partial x^{i}}+u_{i}^{l} \frac{\partial \xi^{j}}{\partial u^{l}}\right) .
$$

From the invariance criterion [2], acting by operator $\underset{1}{X}$ onto equations (10), we get the defining equations. Passing to the manifold (10) we replace $u_{4}^{1}, u_{4}^{2}, u_{4}^{3}, u_{3}^{3}, u_{3}^{4}$ with the remaining variables. Splitting the defining equations with respect to the independent variables, we obtain the coordinates of the operator $X$

$$
\xi^{1}=\left(C_{1}+C_{2}\right) x^{1}+C_{3} x^{2}+f_{1}\left(x^{4}\right), \quad \xi^{2}=\left(C_{1}+C_{2}\right) x^{2}-C_{3} x^{1}+f_{2}\left(x^{4}\right),
$$




$$
\begin{gathered}
\xi^{3}=C_{2} x^{3}+C_{4} x^{4}+C_{5}, \quad \xi^{4}=C_{2} x^{4}+C_{6}, \\
\eta^{1}=C_{1} u^{1}+C_{3} u^{2}+f_{1}^{\prime}\left(x^{4}\right), \quad \eta^{2}=C_{1} u^{2}-C_{3} u^{1}+f_{2}^{\prime}\left(x^{4}\right), \quad \eta^{3}=C_{4}, \\
\eta^{4}=2 C_{1} u^{4}-x^{1} f_{1}^{\prime \prime}\left(x^{4}\right)-x^{2} f_{2}^{\prime \prime}\left(x^{4}\right)+h\left(x^{4}\right),
\end{gathered}
$$

where $C_{1}, \ldots, C_{6}$ are constant, $f_{1}\left(x^{4}\right), f_{2}\left(x^{4}\right), h\left(x^{4}\right)$ are arbitrary functions.

Assuming successively the constants and functions to be non-zero, we find the basis of admissible operators. In Cartesian coordinates the basis of operators for the system (4) is as follows

$$
\begin{gathered}
X_{1}=x \frac{\partial}{\partial x}+y \frac{\partial}{\partial y}+u \frac{\partial}{\partial u}+v \frac{\partial}{\partial v}+2 q \frac{\partial}{\partial q}, \quad X_{2}=x \frac{\partial}{\partial x}+y \frac{\partial}{\partial y}+z \frac{\partial}{\partial z}+t \frac{\partial}{\partial t}, \\
X_{3}=y \frac{\partial}{\partial x}-x \frac{\partial}{\partial y}+v \frac{\partial}{\partial u}-u \frac{\partial}{\partial v}, \quad X_{4}=t \frac{\partial}{\partial z}+\frac{\partial}{\partial w}, \quad X_{5}=\frac{\partial}{\partial z}, \quad X_{6}=\frac{\partial}{\partial t}, \\
X_{7}\left(f_{1}\right)=f_{1}(t) \frac{\partial}{\partial x}+f_{1}^{\prime}(t) \frac{\partial}{\partial u}-x f_{1}^{\prime \prime}(t) \frac{\partial}{\partial q}, \quad X_{8}\left(f_{2}\right)=f_{2}(t) \frac{\partial}{\partial y}+f_{2}^{\prime}(t) \frac{\partial}{\partial v}-y f_{2}^{\prime \prime}(t) \frac{\partial}{\partial q}, \\
X_{9}(h)=h(t) \frac{\partial}{\partial q} .
\end{gathered}
$$

The first two operators are responsible for dilatation transformations, the third one for rotation in the plane $(x ; y)$, the fourth and fifth operators for the Galilean transformation and translation along the axis $z$, the sixth for translation along the $t$-axis. The last three operators contain three arbitrary time-dependent functions and define an infinite-dimensional part of the Lie algebra of the admissible operators.

A similar group analysis was performed for the equations with the long wave approximation in [3]. The model equations were considered in modified variables that take into account the depth of the fluid layer.

In cylindrical coordinates the operators (11) can be written down as follows

$$
\begin{gathered}
\bar{X}_{1}=r \frac{\partial}{\partial r}+\bar{u} \frac{\partial}{\partial \bar{u}}+\bar{v} \frac{\partial}{\partial \bar{v}}+2 q \frac{\partial}{\partial q}, \quad \bar{X}_{2}=r \frac{\partial}{\partial r}+z \frac{\partial}{\partial z}+t \frac{\partial}{\partial t}, \\
\bar{X}_{3}=\frac{\partial}{\partial \theta}, \quad \bar{X}_{4}=t \frac{\partial}{\partial z}+\frac{\partial}{\partial \bar{w}}, \quad \bar{X}_{5}=\frac{\partial}{\partial z}, \quad \bar{X}_{6}=\frac{\partial}{\partial t}, \quad \bar{X}_{9}=h(t) \frac{\partial}{\partial q}, \\
\bar{X}_{7}\left(f_{1}\right)=f_{1}(t) \cos \theta \frac{\partial}{\partial r}-f_{1}(t) \frac{\sin \theta}{r} \frac{\partial}{\partial \theta}+\left(-f_{1}(t) \frac{\sin \theta}{r} \bar{v}+f_{1}^{\prime}(t) \cos \theta\right) \frac{\partial}{\partial \bar{u}}+ \\
+\left(f_{1}(t) \frac{\sin \theta}{r} \bar{u}-f_{1}^{\prime}(t) \sin \theta\right) \frac{\partial}{\partial \bar{v}}-f_{1}^{\prime \prime}(t) r \cos \theta \frac{\partial}{\partial q}, \\
\bar{X}_{8}\left(f_{2}\right)=f_{2}(t) \sin \theta \frac{\partial}{\partial r}+f_{2}(t) \frac{\cos \theta}{r} \frac{\partial}{\partial \theta}+\left(f_{2}(t) \frac{\cos \theta}{r} \bar{v}+f_{2}^{\prime}(t) \sin \theta\right) \frac{\partial}{\partial \bar{u}}+ \\
+\left(-f_{2}(t) \frac{\cos \theta}{r} \bar{u}+f_{2}^{\prime}(t) \cos \theta\right) \frac{\partial}{\partial \bar{v}}-f_{2}^{\prime \prime}(t) r \sin \theta \frac{\partial}{\partial q} .
\end{gathered}
$$

Calculations show that the Lie algebra of the approximate model (9) is

$$
\begin{gathered}
Y_{1}=\partial_{t}, \quad Y_{2}=y \partial_{x}-x \partial_{y}+v \partial_{u}-u \partial_{v}, \quad Y_{3}=-t \partial_{t}+u \partial_{u}+v \partial_{v}+w \partial_{w}+2 q \partial_{q} \\
Y_{4}=z \partial_{z}+w \partial_{w}, \quad Y_{5}=f(x, y, t) \partial_{z}+\left(f_{x}^{\prime} u+f_{y}^{\prime} v+f_{t}^{\prime}\right) \partial_{w} \\
Y_{6}=f_{1}(t) \partial_{x}+f_{1}^{\prime} \partial_{u}-x f_{1}^{\prime \prime} \partial_{q}, \quad Y_{7}=f_{2}(t) \partial_{y}+f_{2}^{\prime} \partial_{v}-y f_{2}^{\prime \prime} \partial_{q} \\
Y_{8}=2 h(t) \partial_{t}+h^{\prime}\left(x \partial_{x}+y \partial_{y}-2 z \partial_{z}\right)+\left(-h^{\prime} u+h^{\prime \prime} x\right) \partial_{u}+\left(-h^{\prime} v+h^{\prime \prime} y\right) \partial_{v}- \\
-\left(4 h^{\prime} w+2 h^{\prime \prime} z\right) \partial_{w}-\left(2 h^{\prime} q+\frac{x^{2}+y^{2}}{2} h^{\prime \prime \prime}\right) \partial_{q}, \quad Y_{9}=\varphi(t) \partial_{q}
\end{gathered}
$$


where $f(x, y, t), f_{1}(t), f_{2}(t), h(t), \varphi(t)$ are arbitrary functions.

In the stationary case when the functions do not depend on time the Lie algebra of admissible operators for equations (9) has the form

$$
\begin{gathered}
\partial_{x}, \partial_{y}, \partial_{q}, z \partial_{z}+w \partial_{w}, u \partial_{u}+v \partial_{v}+w \partial_{w}+2 q \partial_{q}, x \partial_{x}+y \partial_{y}-w \partial_{w}, \\
y \partial_{x}-x \partial_{y}+v \partial_{u}-u \partial_{v}, f(x, y) \partial_{z}+\left(f_{x}^{\prime} u+f_{y}^{\prime} v\right) \partial_{w} .
\end{gathered}
$$

\section{Exact solutions}

Example 1. Let us find a solution to (9) for the operators $\left\langle Y_{5}, Y_{7}\right\rangle$ from the basis (13) with $f=f(x, t), f_{2} \equiv 1$. The invariants of the operators are $\left\{x, t, u, v, q, w f(x, t)-\left(u f_{x}^{\prime}+f_{t}^{\prime}\right) z\right\}$. Hence an invariant solution should be of the form

$$
(u, v, w, q)=\left(U(x, t), V(x, t), W(x, t)+\left(U(x, t) f_{x}^{\prime} / f+f_{t}^{\prime} / f\right) z, Q(x, t)\right) .
$$

The system of equations (9) transforms into the factor system

$$
U_{t}+U U_{x}+Q_{x}=0, \quad V_{t}+U V_{x}=0, \quad U_{x}+U \frac{f_{x}^{\prime}}{f}+\frac{f_{t}^{\prime}}{f}=0 .
$$

The last equation is integrated to give

$$
U=\frac{1}{f(x, t)}\left(-\int f_{t} d x+\varphi(t)\right)
$$

with an arbitrary function $\varphi(t)$. The functions $V(x, t)$ and $Q(x, t)$ are determined from the first two equations of the system (15).

Suppose that $f=f(x)$ does not depend on time $t$, in which case we get the following solution of equations

$$
\begin{gathered}
u=U=\frac{\Phi^{\prime}(t)}{F^{\prime}(x)} ; \quad v=V[F(x)-\Phi(t)] ; \quad w=\frac{\Phi^{\prime}(t) F^{\prime \prime}(x)}{\left(F^{\prime}(x)\right)^{2}} z+W(x, t) ; \\
q=Q=-\int \frac{\varphi^{\prime}(t)}{f(x)} d x-\frac{1}{2}\left(\frac{\Phi^{\prime}(t)}{F^{\prime}(x)}\right)^{2}+\psi(t)
\end{gathered}
$$

with arbitrary functions $\Phi(t), F(x)$ and $f(x)=F^{\prime}(x)$; functions $V$ and $W$ are arbitrary as well. From equation (7) we derive the function that defines the free boundary

$$
\eta(x, y, t)=\frac{1}{g}\left(Q(x, t)-p_{a}(x, y, t)\right)
$$

on which the kinematic condition (6) is met

$$
\eta_{t}+U(x, t) \eta_{x}+V(x, t) \eta_{y}=\frac{\Phi^{\prime}(t) F^{\prime \prime}(x)}{\left(F^{\prime}(x)\right)^{2}} \eta+W(x, t) .
$$

Substitute the relations (16) and (17) into (18) to obtain a condition for $p_{a}(x, y, t)$

$$
p_{a_{t}}+U p_{a_{x}}+V p_{a_{y}}-\frac{\Phi^{\prime} F^{\prime \prime}}{\left(F^{\prime}\right)^{2}} p_{a}=Q_{t}+U Q_{x}-g W .
$$

The function $W(x, t)$ is arbitrary, this implies that we may equate the right hand side of (19) to zero. Then the equation (19) can be integrated with respect to $p_{a}$ to give

$$
p_{a}(x, y, t)=F^{\prime}(x) \cdot P(F(x)-\Phi(t), y-(F(x)-\Phi(t)) t)
$$


with an arbitrary function $P$ of two arguments.

Example 2. Let us find a solution to the system of equations (8) for the operators $\left\langle\bar{X}_{3}, \bar{X}_{4}\right\rangle$ from the basis (12). The invariants of the operators are $\{r, t, \bar{u}, \bar{v}, \bar{w}-z / t, q\}$, so the invariant solution should be of the form

$$
(\bar{u}, \bar{v}, \bar{w}, q)=\left(U(r, t), V(r, t), \frac{z}{t}+W(r, t), q(r, t)\right) .
$$

The system of equations (8) transforms into the factor system

$$
\begin{aligned}
U_{t}+U U_{r}-\frac{1}{r} V^{2}+q_{r} & =0, \quad V_{t}+U V_{r}+\frac{1}{r} U V=0, \\
W_{t}+U W_{r}+\frac{1}{t} W & =0, \quad U_{r}+\frac{1}{t}+\frac{1}{r} U=0 .
\end{aligned}
$$

The equations (20) are integrated starting from the last equation,

$$
\begin{gathered}
U=-\frac{r}{2 t}+\frac{\varphi(t)}{r}, \quad V=\frac{1}{r} F(\lambda), \quad W=\frac{1}{t} G(\lambda), \\
q=\int\left(-U_{t}-U U_{r}+\frac{1}{r} V^{2}\right) d r+\psi(t)=-\frac{3 r^{2}}{8 t^{2}}-\varphi^{\prime}(t) \ln r-\frac{\varphi^{2}(t)}{2 r^{2}}+\int \frac{1}{r^{2}} F^{2}(\lambda) d r+\psi(t),
\end{gathered}
$$

where $\varphi(t), \psi(t), F(\lambda), G(\lambda)$ are arbitrary functions, $\lambda=t r^{2}-2 \int t \varphi(t) d t$.

The kinematic condition (6) in cylindrical coordinates has the form

$$
\frac{\partial \eta}{\partial t}+\bar{u} \frac{\partial \eta}{\partial r}+\bar{v} \frac{1}{r} \frac{\partial \eta}{\partial \theta}-\bar{w}=0 .
$$

From equation (7) in cylindrical coordinates we find

$$
p_{a}(r, \theta, t)=q(r, \theta, t)-g \eta(r, \theta, t)
$$

Suppose that $\eta=\eta(r, t), \partial \eta / \partial \theta=0$. By substituting (21) into (22), we get a solution $\eta(r, t)=t H(\lambda)-G(\lambda)$ with an arbitrary function $H(\lambda)$. From (23) we derive the external pressure on the free surface

$p_{a}(r, t)=q(r, t)-g \eta(r, t)=-\frac{3 r^{2}}{8 t^{2}}-\varphi^{\prime}(t) \ln r-\frac{\varphi^{2}(t)}{2 r^{2}}+\int \frac{1}{r^{2}} F^{2}(\lambda) d r+\psi(t)-g(t H(\lambda)-G(\lambda))$.

Example 3. We find the solution to equation (8) for the following two operators $\left\langle\bar{X}_{1}, \bar{X}_{3}\right\rangle$ from the basis (12). The invariants of the operators are $\left\{z, t, \bar{u} / r, \bar{v} / r, \bar{w}, q / r^{2}\right\}$. The invariant solution is found in the following form

$$
(\bar{u}, \bar{v}, \bar{w}, q)=\left(r U(z, t), r V(z, t), W(z, t),-r^{2} Q(t)\right) .
$$

By inserting it into equation (8), we arrive at the factor system

$$
\begin{gathered}
U_{t}+U^{2}+W U_{z}-V^{2}-2 Q=0, \quad V_{t}+W V_{z}+2 U V=0 \\
W_{t}+W W_{z}=0, \quad 2 U+W_{z}=0
\end{gathered}
$$

From the third equation of the system (25) we find an implicit representation of the solution $W=\Phi(z-t W)$ with an arbitrary function $\Phi(\mu), \mu=z-t W$. The remaining equations of the system (25) define the functions

$$
U=-\frac{1}{2} W_{z}, \quad V=\frac{R}{W_{z}}, \quad Q=-\frac{1}{4} W_{z t}+\frac{1}{8}\left(W_{z}\right)^{2}-\frac{1}{4} W W_{z z}-\left(\frac{R}{W_{z}}\right)^{2}
$$


where $R=R(W)$ is an arbitrary function. The second equation follows from the third one.

Assume that the function $\Phi(\mu)=\alpha \mu+\beta$ is linear, $\alpha, \beta$ are constant. Then from (26) we see that

$$
W=\frac{\alpha z+\beta}{1+\alpha t}, \quad U=-\frac{\alpha}{2(1+\alpha t)}, \quad V=\frac{R_{0}(1+\alpha t)}{\alpha}, \quad Q=\frac{1}{8}\left[\frac{3 \alpha^{2}}{(1+\alpha t)^{2}}-\frac{4 R_{0}^{2}(1+\alpha t)^{2}}{\alpha^{2}}\right] .
$$

Here $\alpha>0$, also it is taken into consideration that the function $Q$ depends only on $t$, therefore $R=R_{0}$ is constant. Thus, we have the exact solution $(\bar{u}, \bar{v}, \bar{w}, q)$ of equations (8).

The kinematic condition (22) in cylindrical coordinates in the given situation has the following form

$$
\frac{\partial \eta}{\partial t}-\frac{r \alpha}{2(1+\alpha t)} \frac{\partial \eta}{\partial r}+\frac{r R_{0}(1+\alpha t)}{\alpha} \frac{\partial \eta}{\partial \theta}=\frac{\alpha z+\beta}{1+\alpha t} .
$$

The solution to that equation is the function

$$
\eta(r, \theta, t)=-\frac{\beta}{\alpha}+\frac{1+\alpha t}{\alpha} F\left(r \sqrt{1+\alpha t}, \quad \theta-\frac{2 r R_{0}(1+\alpha t)^{2}}{3 \alpha^{2}}\right),
$$

which describes the free surface of the fluid $z=\eta(r, \theta, t)$. The function $F$ depends on two arguments and is arbitrary. The external pressure on a free surface is determined by formula (23): $p_{a}(r, \theta, t)=q(r, t)-g \eta(r, \theta, t)$ or

$$
p_{a}=-\frac{r^{2}}{8}\left[\frac{3 \alpha^{2}}{(1+\alpha t)^{2}}-\frac{4 R_{0}^{2}(1+\alpha t)^{2}}{\alpha^{2}}\right]-g\left[-\frac{\beta}{\alpha}+\frac{1+\alpha t}{\alpha} F\left(r \sqrt{1+\alpha t}, \theta-\frac{2 r R_{0}(1+\alpha t)^{2}}{3 \alpha^{2}}\right)\right],
$$

If $\alpha=0$ then $W=\beta$ is constant. In this case the solution to equations $W=\beta=$ const are the following functions

$$
\bar{u}=0, \quad \bar{v}=r V_{0}, \quad \bar{w}=\beta, \quad q=\frac{r^{4}}{2} V_{0}^{2}, \quad V_{0}=\text { const. }
$$

From the kinematic conditions (22) we find the function of the free surface $\eta=\beta t+F\left(r, \theta-V_{0} t\right)$ with an arbitrary function $F$, and from (23) we determine the external pressure on the free surface

$$
p_{a}(r, \theta, t)=q(r, t)-g \eta(r, \theta, t)=\frac{r^{4}}{2} V_{0}^{2}-g\left(\beta t+F\left(r, \theta-V_{0} t\right) .\right.
$$

For the system of equations $(25)$ we introduce the Lagrangian coordinates $(z, t) \rightarrow(\zeta, t)$ in such a manner that

$$
\frac{d z}{d t}=W(z, t),\left.\quad z\right|_{t=0}=\zeta
$$

Denote

$$
W=W(z(\zeta, t), t)=\stackrel{\circ}{W}(\zeta, t) ; V=V(z(\zeta, t), t)=\stackrel{\circ}{V}(\zeta, t) ; U=U(z(\zeta, t), t)=\stackrel{\circ}{U}(\zeta, t) .
$$

From the third equation of the system $(25)$ it does follow that $\stackrel{\circ}{W}(\zeta, t)=W_{0}(\zeta)$, where $W_{0}$ is the value of $\stackrel{\circ}{W}$ for $t=0$, then $z=W_{0} t+\zeta, z_{\zeta}=1+t W_{0}^{\prime}$. From these calculations we see that $W_{z}=W_{0}^{\prime} /\left(1+t W_{0}^{\prime}\right)$. We find that

$$
\begin{gathered}
\stackrel{\circ}{U}=\frac{W_{0}^{\prime}}{2\left(1+t W_{0}^{\prime}\right)}, \quad \stackrel{\circ}{V}=\left(1+t W_{0}^{\prime}\right) V_{0}(\zeta), \quad \stackrel{\circ}{W}=W_{0}(\zeta), \\
\stackrel{\circ}{Q}=\stackrel{\circ}{U_{t}}+\stackrel{\circ}{U^{2}}-\stackrel{\circ}{V}^{2}=\frac{3}{4} \frac{W_{0}^{\prime}}{\left(1+t W_{0}^{\prime}\right)^{2}}-V_{0}^{2}(\zeta),
\end{gathered}
$$


where $V_{0}(\zeta)$ is the value of $\stackrel{\circ}{V}(\zeta, t)$ for $t=0$. Since $\stackrel{\circ}{Q}=\stackrel{\circ}{Q}(t)$ does not depend on $\zeta, W_{0}^{\prime}=\alpha=$ const, $V_{0}=R_{0} / \alpha=$ const, and

$$
\stackrel{\circ}{Q}(t)=\frac{3 \alpha}{8(1+\alpha t)^{2}}-\frac{R_{0}^{2}(1+\alpha t)^{2}}{2 \alpha^{2}}, \stackrel{\circ}{V}=\frac{R_{0}(1+\alpha t)}{\alpha}, \stackrel{\circ}{U}=-\frac{\alpha}{2(1+\alpha t)}, \quad \stackrel{\circ}{W}=\alpha \zeta+\beta,
$$

where $\alpha$ and $\beta$ are constants. The solution (28) in Lagrangian coordinates coincides with the solution in Euler coordinates. The solution (28) shows that the system of equations (25) has no other solutions besides (27).

The author is grateful to Professor V. K. Andreev for useful discussions.

\title{
References
}

[1] K.F.Bowden, Physical oceanography of coastal waters, Ellis Horwood Limited Publishers, New York, Halsted Press, 1983.

[2] L.V.Ovsjannikov, Group analysis of differential equations, Moscow, Nauka, 1978 (in Russian).

[3] A.A.Chesnokov, Symmetries and exact solutions of equations for shallow water of space translation flow, Zhurnal prikladnoi mehaniki i tehnicheskoi fiziki, 49(2008), no. 5, 41-54 (in Russian).

\section{Гидростатическая модель идеальной жидкости, групповые свойства уравнений и их решения}

\section{Александр А. Родионов}

\begin{abstract}
Исследованы групповые свойства уравнений гидростатической модели движения слоя идеальной жидкости относительно функции, определяющей свободную поверхность и толщину слоя жидкости под свободной границей. Даны примеры нескольких точных решений в декартовых и цилиндрических координатах. Они определяют свободную поверхность и давление на этой поверхности.
\end{abstract}

Ключевые слова: идеальная жидкость, гидростатическая модель, групповой анализ, точные решения. 